\title{
openheart Clinical presentation of CIED infection following initial implant versus reoperation for generator change or lead addition
}

\author{
Mariko W Harper, ${ }^{1}$ Daniel Z Uslan, ${ }^{2}$ Arnold J Greenspon, ${ }^{3}$ Larry M Baddour, ${ }^{4}$ \\ Roger G Carrillo, ${ }^{5}$ Stephan B Danik, ${ }^{6}$ Jose M Tolosana, ${ }^{7}$ Katherine Le, ${ }^{4}$ \\ Jose M Miro, ${ }^{7}$ Christoph K Naber, ${ }^{8}$ James Peacock, ${ }^{9}$ Muhammad Rizwan Sohail, ${ }^{4}$ \\ Holenarasipur R Vikram, ${ }^{10}$ Jordan M Prutkin, ${ }^{1}$ for the MEDIC Investigators
}

To cite: Harper MW, Uslan DZ, Greenspon AJ, et al. Clinical presentation of CIED infection following initial implant versus reoperation for generator change or lead addition. Open Heart 2018;5:e000681. doi:10.1136/

openhrt-2017-000681

Received 21 June 2017 Revised 1 February 2018 Accepted 6 March 2018

\section{Check for updates}

For numbered affiliations see end of article.

\section{Correspondence to} Dr Jordan M Prutkin; jprutkin@ cardiology.washington.edu

\section{ABSTRACT}

Objective To explore differences in clinical manifestations and outcomes in those patients who develop infection after undergoing initial implantation versus reoperation. Methods We compared cases of cardiac implantable electronic device (CIED) infection based on initial implantation versus reoperation from 11 centres.

Results There were 432 patients with CIED infection, 178 occurring after initial device placement and 254 after repeat reoperation. No differences were seen in age, sex or device type. Those with infection after initial implant had a higher Charlson Comorbidity Score (median 3 (IQR 2-6) vs 2 (IQR 1-4), p<0.001), shorter time since last procedure (median 8.9 months (IQR $0.9-33.3$ ) vs 19.5 months (IQR 1.1-62.9), $\mathrm{p}<0.0001$ ) and fewer leads $(2.0 \pm 0.6 v s 2.5 \pm 0.9, p<0.001)$. Pocket infections were more likely to occur after a reoperation $(70.1 \%$ vs $48.9 \%$, $\mathrm{p}<0.001)$ and coagulase negative staphylococci (CoNS) was the most frequently isolated organism in this group $(p=0.029)$. In contrast, initial implant infections were more likely to present with higher white cell count $(10.5 \pm 5.1 \mathrm{~g} / \mathrm{dL}$ vs $9.5 \pm 5.4 \mathrm{~g} / \mathrm{dL}, \mathrm{p}=0.025)$, metastatic foci of infection $(16.9 \% v s 8.7 \%, p=0.016)$ and sepsis $(30.9 \%$ vs $19.3 \%, p=0.006)$. There were no differences in in-hospital $(7.9 \%$ vs $5.2 \%, p=0.31)$ or 6 -month mortality (21.9\%vs14.0\%, $\mathrm{p}=0.056)$.

Conclusions CIED infections after initial device implant occur earlier, more aggressively, and often due to Staphylococcus aureus. In contrast, CIED infections after reoperation occur later, are due to CoNS, and have more indolent manifestations with primary localisation to the pocket.

\section{INTRODUCTION}

Cardiac implantable electronic device (CIED) infections have been increasing at a rate greater than the rate of CIED implantation. ${ }^{1-4}$ This increase is predominantly driven by the implantation of CIEDs in an increasingly complex patient population with numerous medical comorbidities. ${ }^{2}$ Prior studies have shown that the risk of

\section{Key questions}

What is already known about this subject?

- The global incidence of cardiac implantable electronic device (CIED) infections is now increasing out of proportion to the rate of device implantation, driven predominantly by the implantation of devices in patients with increasing complexity and medical comorbidities. Prior studies have shown that the risk of developing CIED infection is higher after a generator change compared with initial device implant but patient differences between CIED infections after initial implantation versus reoperation have not been previously described.

What does this study add?

- To the best of our knowledge, this study using the Multicenter Electrophysiologic Device Infection Cohort cohort, the largest registry of CIED infections, is the first to compare differences in patient characteristics, clinical manifestations and outcomes in patients who develop CIED infections after an initial (de novo) device implantation versus a device reoperation (such as a revision or generator change).

How might this impact on clinical practice?

- Our study found that patients with CIED infection occurring after initial device implantation tended to present earlier, with more severe clinical manifestations of infection and more often due to Staphylococcus aureus, whereas those with a reoperation presented later with the more indolent coagulase negative staphylococcus. Our study makes important contributions to our current understanding of CIED infections and highlights the need for providers to recognise that CIED infection may present in different ways based on last procedure type.

CIED infection is higher after a generator change compared with the initial device implantation. ${ }^{4-8}$ It is hypothesised that this 
greater rate of infection is due to higher incidence of CIED pocket colonisation after repeat device reoperations. Up to $42 \%$ of CIED pockets are thought to be colonised by microorganisms, although only a fraction of those ostensibly colonised eventuate in clinical infection. $^{56}$

It remains unknown if there are differences in the patient characteristics, clinical manifestations and time to infection onset between those who have CIED infection after an initial implantation versus after a reoperation for generator change or lead addition and if clinical outcomes are different between these two groups. The purpose of this study was to examine the differences in patient characteristics, presentation and outcomes in device infections after initial (de novo) implantation versus repeat reoperation.

\section{METHODS \\ Subjects}

The Multicenter Electrophysiologic Device Infection Cohort (MEDIC) was a prospective registry of patients with CIED infections at 11 international sites. All subjects were 18 years of age or older and provided informed consent. Enrolment was from 1 January 2009 until 31 December 2012. Subjects were enrolled at the time of consent and were followed for 6 months after the time of study enrolment. Patient demographics, medical history, medication use, presentation, laboratory and radiographic data and treatment were recorded. The Charlson Comorbidity index, obtained using a weighted average of 19 different comorbidity categories, was used to determine comorbidity severity.

At least two sets of blood cultures were obtained from all patients at initial presentation. Gram stain and cultures from device lead and generator pocket specimens were obtained in patients who underwent device removal. Participants received antibiotics guided by culture results and the antibiotic susceptibility data of causative organisms in accordance with published guidelines. ${ }^{7}$ Reimplantation was performed based on the treating physician's discretion.

Subjects were divided into those who developed an infection after initial implant and those who developed infection after reoperation. Reoperation was defined as accessing a CIED pocket for the purpose of a generator change, pocket revision, lead addition, lead revision or lead extraction. There was no clinical suspicion for CIED infection at the time of the repeat reoperation for any of the subjects in the infection after re-operation group.

Subjects were included regardless of the treatment received for the infection. While most subjects underwent percutaneous CIED system extraction, patients could also be treated with open surgical device removal or chronic suppressive antibiotic therapy without CIED removal based on patient and physician's discretion. In cases of percutaneous CIED removal, the extraction tools used were recorded.

\section{Definitions}

A pocket infection was defined as localised pocket warmth, tenderness, erythema, fluctuance, purulent drainage, wound dehiscence and/or erosion of the generator or leads at the site of CIED. Lead-associated endocarditis (LAE) was defined using the modified Duke criteria. ${ }^{8}$ This category included persistent bloodstream infection with positive blood cultures and the presence of a vegetation on the lead seen on transthoracic or transoesophageal echocardiography. Vegetations were defined as oscillating intracardiac masses attached to a pacemaker or implantable cardioverter-defibrillator (ICD) lead seen in two or more echocardiographic planes. LAE was also diagnosed in subjects with negative blood cultures if there was evidence of a lead vegetation on echocardiography and clinical signs and/or symptoms consistent with localised pocket infection were present. In addition, cryptogenic blood stream infection in the absence of vegetation was also classified as a CIED infection if the infection resolved after removal of the CIED. Sepsis was defined as the presence of two or more systemic inflammatory response criteria (temperature $>38^{\circ} \mathrm{C}$ or $<36^{\circ} \mathrm{C}$, heart rate $>90$, respiratory rate $>20$ or $\mathrm{pCO}_{2}<32 \mathrm{~mm} \mathrm{Hg}$ or white cell count $>12$ or $<4 \times 10^{\wedge} 9 / \mathrm{L}$ ) in tandem with clinical suspicion for infection on the part of the enrolling clinician.

Patients were followed for 6 months after study enrolment and data including the subject's vital status, new device implantation and total duration of completed antibiotic therapy were documented. This data was obtained both by review of medical records and/or contact with the patient's treating physician.

\section{Statistical analysis}

$\chi^{2}$ test or Fisher's exact test was used to compare categorical variables. Two sample Student's t-test or Wilcoxon ranks sum test used for comparison of differences between groups and comparable non-parametric tests were used, as appropriate. All tests were two-sided, and a $p$ value of $<0.05$ was considered statistically significant. Statistical analyses were completed using SAS V.9.3.

\section{RESULTS}

A total of 432 subjects were included in the analysis. There were 178 subjects in the infection after initial implant group and 254 subjects in the infection after reoperation group. Subject demographics are reported in table 1 . There was no difference in age, gender or race between groups. Subjects in the infection after initial implant had a higher Charlson Comorbidity Score than subjects in the reoperation group (median 3 (IQR 2-6) vs 2 (IQR 1-4), $\mathrm{p}<0.001$ ). They were also more likely to have had a solid organ transplant $(2.8 \%$ vs $0 \%, \mathrm{p}=0.011)$ and be on immunosuppressive medications $(10.1 \%$ vs $4.3 \%$, $\mathrm{p}=0.03)$. There were no other significant differences in patient characteristics between the two groups. 


\begin{tabular}{|c|c|c|c|}
\hline & $\begin{array}{l}\text { Initial } \\
\text { implantation } \\
(n=178)\end{array}$ & $\begin{array}{l}\text { Reoperation } \\
(n=254)\end{array}$ & $P$ values \\
\hline Age (SD) & $67.9(14.0)$ & $67.8(14.9)$ & 0.905 \\
\hline Male sex & 136 (76.4) & 202 (79.5) & 0.478 \\
\hline Race/ethnicity & & & 0.266 \\
\hline White/Caucasian & $152(85.4)$ & $208(81.9)$ & \\
\hline Black & $19(7.5)$ & $13(7.3)$ & \\
\hline Asian & $1(0.6)$ & $0(0)$ & \\
\hline Hispanic & $8(4.5)$ & $22(8.7)$ & \\
\hline
\end{tabular}

Type of device

\begin{tabular}{|c|c|c|c|}
\hline PM & $125(49.2)$ & $96(53.9)$ & 0.344 \\
\hline ICD & 75 (29.5) & $54(30.3)$ & \\
\hline CRT & $54(21.3)$ & $28(15.7)$ & \\
\hline $\begin{array}{l}\text { History of intravenous } \\
\text { drug abuse }\end{array}$ & $3(1.7)$ & $8(3.1)$ & 0.537 \\
\hline $\begin{array}{l}\text { Previous history of CIED } \\
\text { infection }\end{array}$ & $15(8.4)$ & $34(13.4)$ & 0.124 \\
\hline Haemodialysis & $21(11.8)$ & $16(6.3)$ & 0.054 \\
\hline $\begin{array}{l}\text { Indwelling central } \\
\text { venous catheter }\end{array}$ & $7(3.9)$ & $4(1.6)$ & 0.172 \\
\hline $\begin{array}{l}\text { Immunosuppressive } \\
\text { drug use within past } \\
6 \text { months }\end{array}$ & $18(10.1)$ & $11(4.3)$ & 0.03 \\
\hline $\begin{array}{l}\text { Steroid use within the } \\
\text { past } 30 \text { days }\end{array}$ & $8(4.5)$ & $10(3.4)$ & 0.81 \\
\hline $\begin{array}{l}\text { Solid organ transplant } \\
\text { history }\end{array}$ & $5(2.8)$ & $0(0)$ & 0.011 \\
\hline Coronary artery disease & $88(49.4)$ & $130(51.3)$ & 0.769 \\
\hline $\begin{array}{l}\text { Coronary artery bypass } \\
\text { graft }\end{array}$ & $42(23.6)$ & $55(21.7)$ & 0.641 \\
\hline $\begin{array}{l}\text { Congenital heart } \\
\text { disease }\end{array}$ & $7(3.9)$ & $17(6.7)$ & 0.287 \\
\hline $\begin{array}{l}\text { History of prosthetic } \\
\text { heart valve }\end{array}$ & $33(18.5)$ & $34(13.4)$ & 0.177 \\
\hline $\begin{array}{l}\text { History of endovascular } \\
\text { graft }\end{array}$ & $19(10.7)$ & $29(11.4)$ & 0.874 \\
\hline Anticoagulation use & $52(39.4)$ & $90(35.4)$ & 0.472 \\
\hline $\begin{array}{l}\text { Charlson Comorbidity } \\
\text { Index Score, median } \\
\text { (IQR) }\end{array}$ & $3(2,6)$ & $2(1,4)$ & $<0.001$ \\
\hline $\begin{array}{l}\text { Mean ejection fraction } \\
\text { (SD) }\end{array}$ & $35.9(16.1)$ & $34.4(14.5)$ & 0.681 \\
\hline
\end{tabular}

CIED, cardiac implantable electronic device; CRT, cardiac resynchronisation therapy; ICD, implantable cardioverterdefibrillator; PM, pacemaker.

There was no difference between the types of CIED (pacemaker vs ICD vs biventricular device) between the two groups. As expected, subjects in the infection after initial implant group had a shorter duration since initial implant (median 21.1 months (IQR 2.9-47.2) vs 71.0 months (IQR 32.5-113.2), $\mathrm{p}<0.001$ ) and fewer total leads $(2.0 \pm 0.6$ vs $2.5 \pm 0.9, \mathrm{p}<0.001)$. Subjects in the infection after initial implant group presented significantly earlier since their last device-related procedure as compared with the reoperation group (median 8.9 months (IQR $0.9-33.3$ ) vs 19.5 months (IQR 1.1-62.9), $\mathrm{p}<0.0001$ ) (figure 1). Although the reoperation group was more likely to have a pocket infection than the initial implant group $(70.1 \%$ vs $48.9 \%, \mathrm{p}<0.001)$, there was no statistically significant difference in the rate of endocarditis ( $47.3 \%$ vs $51.7 \%$, $\mathrm{p}=0.65$ ). Subjects in the infection after initial implant group were more likely to present with more severe markers of infection, including an elevated leucocyte count $\left(10.5 \pm 5.1 \times 10^{\wedge} 9 / \mathrm{L}\right.$ vs $9.5 \pm 5.4 \times 10^{\wedge} 9 / \mathrm{L}$, $\mathrm{p}=0.025)$, sepsis $(30.9 \%$ vs $19.3 \%, \mathrm{p}=0.006)$, new moderate or severe valvular regurgitation $(19.5 \%$ vs $12.3 \%, \mathrm{p}=0.05)$ and metastatic sites of infection $(16.9 \%$ vs $8.7 \%, \mathrm{p}=0.016$ ) (table 2).

Differences in the microorganisms causing infection were also apparent between the two groups. While a variety of microorganisms were isolated, coagulase negative staphylococcus was isolated more frequently in subjects in the infection after reoperation group compared with Staphylococcus aureus or other organisms $(\mathrm{p}=0.029)$. Other organisms included gram-positive organisms such as Propionibacterium acnes, Streptococcus viridans and Enterococcus species, gram negative rods such as Escherischia coli and Klebsiella species and fungi including Candida. A total of 203 subjects developed bacteraemia (47.0\%), 86 in the infection after initial implant group and 117 in the infection after reoperation group. Of those who developed bacteraemia, the source was attributed to the CIED pocket site more frequently in those in the infection after reoperation group compared with the infection after initial implant $(51.9 \%$ vs $24.0 \%$, $\mathrm{p}=0.001$ ).

There was also a difference in the ease of device extraction between the two groups. Subjects in the infection after reoperation group were more likely to need an excimer laser sheath for removal $(65.6 \%$ vs $26.4 \%$, $\mathrm{p}<0.001)$.

Despite the differences in type and severity of CIED infection, there was no difference in in-hospital mortality $(7.9 \%$ vs $5.2 \%, \mathrm{p}=0.31)$ or 6 -month mortality (21.9 vs 14.0, $\mathrm{p}=0.056$ ) between the groups with infections after initial implant and reoperation.

\section{DISCUSSION}

This prospective cohort study demonstrates the distinct differences in those patients with CIED infections that present after an initial device implant versus after repeat reoperation. Subjects in the infection after initial implant group developed infections earlier, manifested more severe clinical presentations, and more often had infections due to $S$. aureus. Subjects in the infection after reoperation group presented almost a year later with clinical presentations that were much more indolent and with 

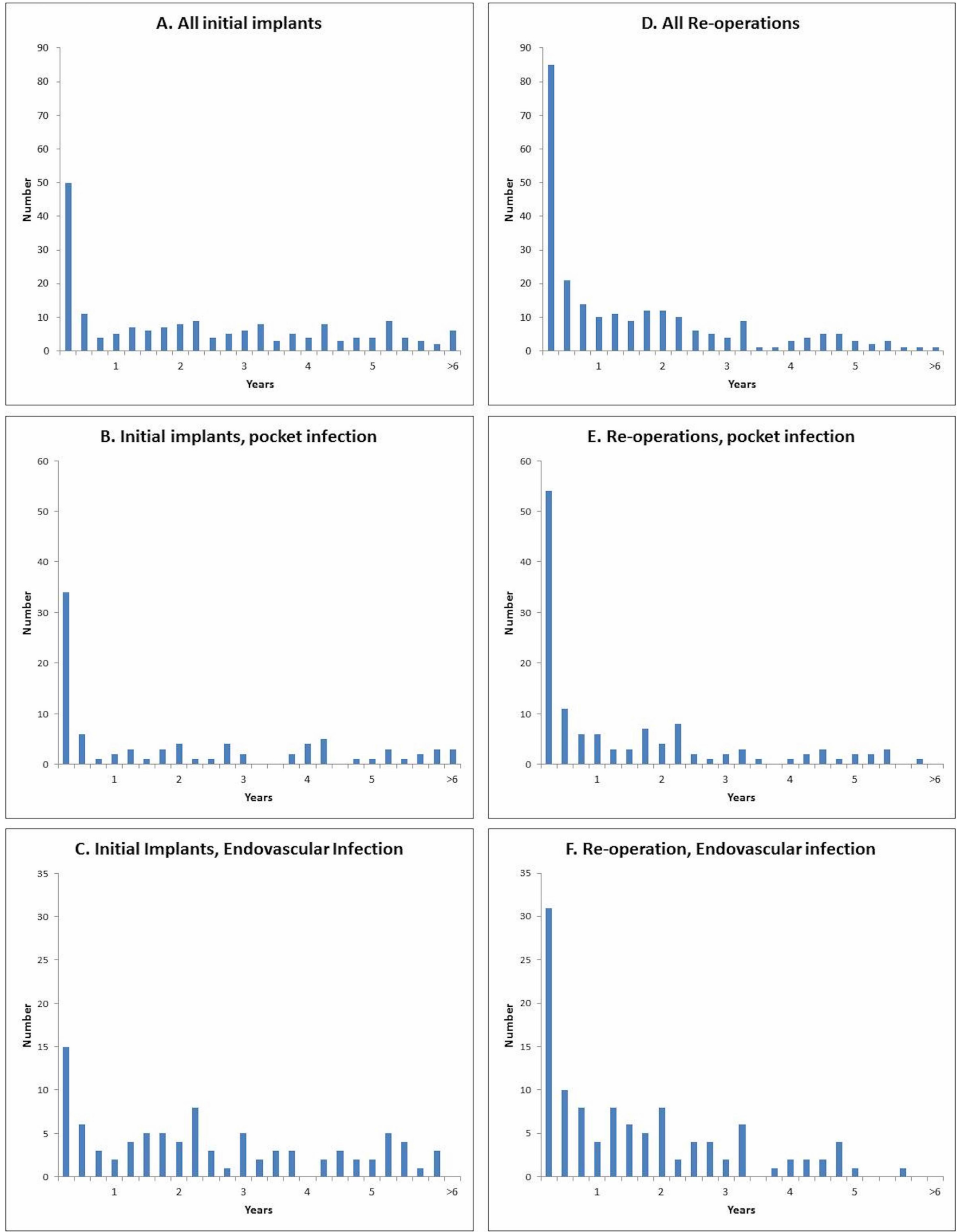

Figure 1 Number of infections from time since last device-related procedure. (A) All initial implants. (B) Initial implants with pocket infections. (C) Initial implants with bacteraemia or lead-associated endocarditis. (D) All reoperations. (E) Reoperations with pocket infections. (F) Reoperations with bacteraemia or lead-associated endocarditis. 
Table 2 Differences between infections after initial implantation and repeat reoperation

\begin{tabular}{llll}
\hline & Initial implantation (n=178) & Reoperation (n=254) & P values \\
\hline Localised pocket infection & $87(48.9 \%)$ & $178(70.1 \%)$ & $<0.001$ \\
\hline Lead-associated endocarditis & $92(51.7 \%)$ & $120(47.3 \%)$ & 0.363 \\
\hline Cryptogenic blood stream infection & $46(25.8 \%)$ & $61(24 \%)$ & 0.734 \\
\hline Total number of leads in place (SD) & $2.0 \pm 0.6$ & $2.5 \pm 0.9$ & $<0.001$ \\
\hline Median implant duration (IQR) & $21.1(2.9,47.2)$ & $71(32.5,113.2)$ & $<0.001$ \\
\hline Median time from last procedure until infection (IQR) & $8.9(0.9,33.3)$ & $19.5(1.1,62.9)$ & $<0.0001$ \\
\hline Excision requiring laser sheath & $47(26.4 \%)$ & $166(65.6 \%)$ & $<0.001$ \\
\hline Fever $>38^{\circ} \mathrm{C}$ & $89(50 \%)$ & $115(45.3 \%)$ & 0.378 \\
\hline New moderate or severe valve regurgitation & $33(19.5 \%)$ & $29(12.3 \%)$ & 0.05 \\
\hline Sepsis & $55(30.9 \%)$ & $49(19.3 \%)$ & 0.006 \\
\hline Pain at pocket site & $58(32.6 \%)$ & $125(49.2 \%)$ & 0.001 \\
\hline Warmth at pocket site & $45(25.3 \%)$ & $91(35.8 \%)$ & 0.021 \\
\hline Pus from pocket site & $35(19.7 \%)$ & $80(31.5 \%)$ & 0.008 \\
\hline Drainage from pocket & $49(27.5 \%)$ & $100(39.4 \%)$ & 0.013 \\
\hline Skin ulceration at pocket site & $17(9.6 \%)$ & $36(14.2 \%)$ & 0.18 \\
\hline Skin erosion at pocket site & $29(16.3 \%)$ & $61(24 \%)$ & 0.055 \\
\hline Metastatic foci of infection & $30(16.9 \%)$ & $22(8.7 \%)$ & 0.016 \\
\hline Stigmata of endocarditis & $4(2.2 \%)$ & $11(4.3 \%)$ & 0.295 \\
\hline Mean C-reactive protein, mg/dL (SD) & $28.3 \pm 37.06$ & $35.8 \pm 39.4$ & 0.1 \\
\hline Mean erythrocyte sedimentation rate, $\mathrm{mm} / \mathrm{hour}$ (SD) & $41.8 \pm 34.1$ & $34.7 \pm 28.0$ & 0.42 \\
\hline Mean leucocyte count (×10^9L) (SD) & $10.5 \pm 5.1$ & $9.5 \pm 5.4$ & 0.025 \\
\hline
\end{tabular}

symptoms and signs more likely localised to the CIED pocket.

\section{Temporal differences in onset of infection}

The late presentation of infection in the reoperation group is of interest as several recent studies using a cut-off of 6 months to define early versus late infection have not shown any difference in the rate of device infections between infection after initial implant and infection after reoperation. ${ }^{9}{ }^{10}$ Those studies may have underestimated the rate of infection by using a breakpoint of 6 months. ${ }^{11}{ }^{12}$ For instance, Sohail et $a l^{10}$ demonstrated that there was no difference in the rate of ICD infection between subjects presenting after initial device implant or repeat reoperation within 6 months, but a difference may have become evident with longer follow-up. In contrast, using this MEDIC registry data, our group did show that there was a significant difference in the clinical presentation of subjects with lead-associated endocarditis who presented early (within 6 months) or late ( $>6$ months) after last follow-up. ${ }^{9}$ The Danish Pacemaker Register examined risk of infections after initial implant and generator change and found that the predominance of infections occurred within the first year, with a higher risk of pocket infection after a repeat procedure, although this risk declined after the first year. ${ }^{13}$ Lekkerker et al also demonstrated that in those with a CIED infection within 1 year of the last device related procedure, that procedure was most like a device revision. ${ }^{6}$ Our data suggest defining early infection onset at less than 6 months, or even 12 months, may introduce a bias of higher infection rates towards infections occurring after initial device placement rather than infection after reoperation.

In the Danish registry, which was limited to pacemakers, risk factors associated with infection included device replacement, male sex, younger age, earlier year of implant, the absence of prophylactic perioperative antibiotics and increased number of pacemaker-related procedures. Our study included subjects with ICDs and cardiac resynchronisation therapy so it may well have represented a different and perhaps more medically complicated patient population.

\section{Differences in clinical presentation between infection after initial implant and after reoperation}

Subjects with infection after initial implant were found to have increased medical comorbidities, as determined by the Charlson Comorbidity Index, and were more likely to have received immunosuppressive therapies and have a history of solid organ transplantation. Patients with infection after initial implant subjects also had a trend towards increased haemodialysis requirements. It can be postulated that the increased comorbidities predispose this group of patients to more severe infectious presentation found in this group, including sepsis, a higher degree of leucocytosis and metastatic foci of infection. 
Unfortunately, as we do not have reliable data about the initial device implantation, we cannot reach any conclusions regarding the clinical circumstances and risk factors present at the time of the last procedure. However, one can postulate that the frequently encountered several month window to prepare for an elective device replacement may allow time to optimise the medical status as best as possible in many patients in the infection after reoperation group. Subjects presenting for initial device placement may not have the same luxury of time due to the acute need for device placement.

\section{Role of microbial pocket colonisation and reactivation}

Most pocket infections are thought to originate from organisms that enter the pocket locally, either at the time of initial device implant or following repeat reoperation, rather than from blood-borne seeding. In fact, more than one-third of CIED pockets are colonised with organisms at the time of generator change and have linked some of these organisms to subsequent clinical infection. ${ }^{61415}$ In our study, we found that while a variety of organisms were seen in both the infection after initial implant and infection after reoperation groups, coagulase negative staphylococcal species were more frequently isolated in the infection after reoperation group, while $S$. aureus was seen more frequently in the infection after initial implant group. The increased presence of $S$. aureus in infection after initial implant may explain the more acute infectious presentation in this group.

As this study was not designed to assess the risks of developing infection after initial implant or infection after reoperation, this question is not addressed by our study. However, several studies have previously shown that there are higher rates of infection after reoperation compared with infection after initial implant, perhaps due to the high prevalence of pocket colonisation in this group. ${ }^{616}$

Established CIED pockets are predominantly fibrous structures with little vascularisation and thus represent an unfavourable environment for developing a robust immune response. Additionally, it is thought that there is a 'race for the surface' between microorganisms and the host tissue response at the time of CIED placement. ${ }^{16}{ }^{17}$ If the host tissue response is able to establish itself first, there is less opportunity for microbial colonisation and infection at the pocket site. In contrast, if the bacteria are able to colonise the surface before tissue healing, there can be impaired clearance of these organisms. Bacterial defences such as the secretion of biofilms are thought to be primarily responsible. ${ }^{17}$ Ultimately, colonised organisms may live in equilibrium with a healthy host and not cause clinical infection. However, when there is an insult to the pocket, such as device reoperation, this event can upset the balance between host and microorganism immune responses and CIED pocket infection may result. Subjects in the reoperation group were more likely to present with localised pocket infection and with less severe systemic signs of infection. This observation is consistent with the assumption that there is reactivation of organisms in the pocket site at the time of repeat reoperation.

To the best of our knowledge, there have been no studies that examine how the microbiome of the pocket may change over time or with each reoperation. In addition, no study has shown that removing the fibrous capsule at the generator pocket site at the time of a repeat reoperation reduces the CIED infection risk. While this approach would presumably reduce the microbial burden within the pocket, the overall infection risk may be the same if not higher due to the increased risk of pocket haematoma, another independent risk factor for CIED infection. ${ }^{12}$

\section{Study limitations}

As this study involved subjects presenting with CIED infection at the time of enrolment in the registry, the data cannot be used to predict those who are at risk for developing CIED infection; these variables have been well described in other studies, however. ${ }^{20}$ This study, despite being multicentre and multinational, was limited to subjects enrolled in tertiary referral centres with the capacity to perform lead extractions and may thus be subject to referral bias.

\section{CONCLUSION}

CIED infections occurring after initial device implant present earlier exhibit more severe clinical manifestations of infection and are more often caused by $S$. aureus. In contrast, CIED infections after repeat reoperation occur later and present more indolently, typically with localised pocket infection due to coagulase negative staphylococci.

\section{Author affiliations}

${ }^{1}$ Department of Medicine, Division of Cardiology, University of Washington, Seattle, Washington, USA

${ }^{2}$ Division of Infectious Diseases, Department of Medicine, UCLA, Los Angeles, California, USA

${ }^{3}$ Division of Cardiology, Department of Medicine, Thomas Jefferson University Hospital, Philadelphia, Pennsylvania, USA

${ }^{4}$ Division of Infectious Diseases, Department of Medicine, Mayo Clinic College of Medicine, Rochester, Minnesota, USA

${ }^{5}$ Division of Cardiothoracic Surgery, University of Miami, Miller School of Medicine, Miami, Florida, USA

${ }^{6}$ Cardiac Arrhythmia Service, Massachusetts General Hospital, Boston,

Massachusetts, USA

${ }^{7}$ Infectious Diseases Service, Hospital Clinic, IDIBAPS, University of Barcelona, Barcelona, Spain

${ }^{8}$ Klinik für Kardiologie und Angiologie, Elisabeth Krankenhaus, Essen, Germany ${ }^{9}$ Section on Infectious Diseases, Department of Medicin, Wake Forest School of Medicine, Winston-Salem, North Carolina, USA

${ }^{10}$ Division of Infectious Diseases, Department of Medicine, Mayo Clinic Arizona, Phoenix, Arizona, USA

Collaborators JMP (University of Washington, Seattle, WA, USA), DZU (UCLA, Los Angeles, CA, USA), AJG (Thomas Jefferson University Hospital, Philadelphia, PA), MRS, LMB, KL (Mayo Clinic College of Medicine, Rochester, MN, USA), RGC (University of Miami, Miami, FL, USA), SBD (Massachusetts General Hospital, Boston, MA, USA), JMT, JMM, S Ninot, A del Rio X Castañeda, F Marco, C García de 
la María, C Cervera, Y Armero, M Almela, C Paré, A Moreno, L Mont and C Mestres (Hospital Clinic, IDIBAPS, University of Barcelona, Barcelona, Spain). Elisabeth Blank, CKN (Elisabeth Krankenhaus, Essen, Germany), JP (Wake Forest School of Medicine, Winston-Salem, NC, USA), HRV (Mayo Clinic, Phoenix, AZ, USA).

Contributors All the authors were actively involved in the planning, conduct and reporting of this original work.

Funding This study was funded, in part, by a grant from the American Heart Association (DZU, Primary Investigator). Data from the Hospital Clinic of Barcelona (Spain) were supported in part by a grant from the 'Ministerio de Sanidad y Consumo', the 'Instituto de Salud Carlos III' and the Spanish Network for the Research in Infectious Diseases (REIPI RD06/0008), Madrid (Spain).

Competing interests None declared.

Patient consent Obtained.

Ethics approval The local Institutional Review Board at each site approved the study protocol.

Provenance and peer review Not commissioned; externally peer reviewed.

Open Access This is an Open Access article distributed in accordance with the Creative Commons Attribution Non Commercial (CC BY-NC 4.0) license, which permits others to distribute, remix, adapt, build upon this work non-commercially, and license their derivative works on different terms, provided the original work is properly cited and the use is non-commercial. See: http://creativecommons.org/ licenses/by-nc/4.0/

(C) Article author(s) (or their employer(s) unless otherwise stated in the text of the article) 2018. All rights reserved. No commercial use is permitted unless otherwise expressly granted.

\section{REFERENCES}

1. Cabell $\mathrm{CH}$, Heidenreich $\mathrm{PA}$, $\mathrm{Chu} \mathrm{VH}$, et al. Increasing rates of cardiac device infections among Medicare beneficiaries: 1990-1999. Am Heart J 2004:147:582-6.

2. Greenspon AJ, Patel JD, Lau E, et al. 16-year trends in the infection burden for pacemakers and implantable cardioverter-defibrillators in the United States 1993 to 2008. J Am Coll Cardiol 2011;58:1001-6.

3. Voigt A, Shalaby A, Saba S. Rising rates of cardiac rhythm management device infections in the United States: 1996 through 2003. J Am Coll Cardiol 2006;48:590-1.

4. Voigt A, Shalaby A, Saba S. Continued rise in rates of cardiovascular implantable electronic device infections in the United States: temporal trends and causative insights. Pacing Clin Electrophysiol 2010;33:414-9.

5. Dy Chua J, Abdul-Karim A, Mawhorter S, et al. The role of swab and tissue culture in the diagnosis of implantable cardiac device infection. Pacing Clin Electrophysiol 2005;28:1276-81.

6. Rohacek M, Weisser M, Kobza R, et al. Bacterial colonization and infection of electrophysiological cardiac devices detected with sonication and swab culture. Circulation 2010;121:1691-7.

7. Baddour LM, Epstein AE, Erickson CC, et al. Update on cardiovascular implantable electronic device infections and their management: a scientific statement from the American Heart Association. Circulation 2010;121:458-77.

8. Li JS, Sexton DJ, Mick N, et al. Proposed modifications to the Duke criteria for the diagnosis of infective endocarditis. Clin Infect Dis 2000;30:633-8.

9. Greenspon AJ, Prutkin JM, Sohail MR, et al. Timing of the most recent device procedure influences the clinical outcome of lead-associated endocarditis results of the MEDIC (Multicenter Electrophysiologic Device Infection Cohort). J Am Coll Cardiol 2012:59:681-7.

10. Sohail MR, Hussain S, Le KY, et al. Risk factors associated with early- versus late-onset implantable cardioverter-defibrillator infections. J Interv Card Electrophysiol 2011;31:171-83.

11. Uslan DZ, Gleva MJ, Warren DK, et al. Cardiovascular implantable electronic device replacement infections and prevention: results from the REPLACE Registry. Pacing Clin Electrophysiol 2012;35.

12. Prutkin JM, Reynolds MR, Bao H, et al. Rates of and factors associated with infection in 200909 medicare implantable cardioverter-defibrillator implants: results from the national cardiovascular data registry. Circulation 2014;130:1037-43.

13. Johansen JB, Jørgensen OD, Møller M, et al. Infection after pacemaker implantation: infection rates and risk factors associated with infection in a population-based cohort study of 46299 consecutive patients. Eur Heart J 2011;32:991-8.

14. Kleemann T, Becker T, Strauss M, et al. Prevalence of bacterial colonization of generator pockets in implantable cardioverter defibrillator patients without signs of infection undergoing generator replacement or lead revision. Europace 2010;12:58-63.

15. Mason PK, Dimarco JP, Ferguson JD, et al. Sonication of explanted cardiac rhythm management devices for the diagnosis of pocket infections and asymptomatic bacterial colonization. Pacing Clin Electrophysiol 2011;34:143-9.

16. Gristina AG. Biomaterial-centered infection: microbial adhesion versus tissue integration. Science 1987;237:1588-95.

17. Subbiahdoss G, Kuijer R, Grijpma DW, et al. Microbial biofilm growth vs. tissue integration: "the race for the surface" experimentally studied. Acta Biomater 2009;5:1399-404. 